\title{
Tangence
}

\section{La problématique du bilinguisme, Franco-Ontarian Style : L'homme invisible/The Invisible Man de Patrice Desbiens}

\section{Marie-Chantal Killeen}

Numéro 56, décembre 1997

Postures scripturaires dans la littérature franco-ontarienne

URI : https://id.erudit.org/iderudit/025959ar

DOI : https://doi.org/10.7202/025959ar

Aller au sommaire du numéro

Éditeur(s)

Tangence

ISSN

0226-9554 (imprimé)

1710-0305 (numérique)

Découvrir la revue

Citer cet article

Killeen, M.-C. (1997). La problématique du bilinguisme, Franco-Ontarian Style : L'homme invisible/The Invisible Man de Patrice Desbiens. Tangence, (56), 80-90. https://doi.org/10.7202/025959ar d'utilisation que vous pouvez consulter en ligne. 


\title{
La problématique du bilinguisme, Franco-Ontarian Style : L'homme invisible/ The Invisible Man de Patrice Desbiens
}

\author{
Marie-Chantal Killeen
}

Écrire, c'est avoir la passion de l'origine.

Edmond Jabès, Le retour au Livre

Étudier le poids relatif des langues qui interviennent dans l'écriture bilingue et s'interroger sur les effets et les sens qui y sont produits, c'est examiner dès lors les divers rapports qui lient l'écrivain à la langue. Il s'agit ou bien d'un rapport de complémentarité (c'est la *bi-langue * d'Abdelkebir Khatibi ${ }^{1}$ ), signe de compétence et d'harmonie langagières, ou bien d'un rapport hiérarchique, qui valorise une langue au détriment de l'autre. Si, dans le meilleur des cas, le métissage linguistique peut enrichir le locuteur en redoublant son potentiel de communiquer, il peut également générer un fort sentiment de dépossession de la langue maternelle. C'est cette dernière possibilité qu'explore l'écrivain Patrice Desbiens, dans une œuvre qui serait, au dire de François Paré, "l'une des plus importantes en Ontario français *2. La pratique scripturaire du bilinguisme dans L'bomme invisible/ The Invisible Man témoigne de la perméabilité d'une langue maternelle non seulement carencée mais foncièrement incapable de fonder un sujet. Car il ne s'agit pas, dans ce récit publié dans les deux langues officielles du Canada (version française sur la page de gauche et version anglaise sur la page de droite), d'une réflexion philosophique sur l'inadéquation du langage à dire l'essence des choses. Pas plus que l'alternance de codes qui structure le texte de Desbiens ne tente de carnavaliser, à la manière ludique d'un Ducharme par exemple, les vocables puisés dans l'autre langue. Non: ici, la ligne de partage entre l'anglais et le français, ainsi que l'interférence des deux langues, ne découlent pas d'un libre choix (comme s'il pouvait en aller autrement, sans

1 Abdelkebir Khatibi, Amour bilingue, Casablanca, EDDIF, 1992.

2 François Paré, Les littératures de l'exiguitê, Ottawa, Le Nordir, 1992, p.128. 
autre forme de procès), encore moins d'une recherche du pittoresque. C'est que ce double récit est écrit deux fois, semble-t-il, dans la langue de l'autre. Il faut entendre, par cette affirmation un peu sibylline, qu'aucune des deux langues ne semble appartenir en propre au protagoniste, Franco-Ontarien écartelé entre deux codes et deux cultures. Dans cet entre-deux, l'homme invisible fait, par le biais du langage - et à cause de lui, faut-il s'empresser d'ajouter -, l'expérience de sa plus profonde aliénation. Dans un entretien à propos de L'bomme invisible/The Invisible Man, Desbiens a souligné les répercussions d'une telle dépossession linguistique: "Dans ce livre je voulais dire qu'être pris entre deux cultures, c'est épouvantable. [...] Toi, tu es coincé entre les deux: tu comprends les deux langues, tu parles les deux langues parfaitement mais tu n'es personne. ${ }^{3}$

Le récit de Desbiens dramatise ainsi jusque dans sa forme hybride la marginalisation de la communauté francophone de Timmins, ville minière du nord de l'Ontario où s'amorce le récit, et dont la communauté francophone, à l'instar d'autres communautés franco-ontariennes, est menacée par la force hégémonique de l'assimilation et minée par des taux de chômage et d'analphabétisme chroniques. Une disjonction fondamentale se donne à voir entre la représentation d'une Amérique aussi opaque que l'homme est invisible, et la réalité de Timmins, comme en témoigne cette allusion à un -orchestre de rock and roll local [qui joue] California Dreamin' tandis que dehors une tempête de neige mange la lumière " 4 . Par ailleurs, le récit fait état de la difficulté tout aussi irréductible de faire entrer la langue des FrancoOntariens dans le jeu de la représentation, et par là même dans la sphère du pouvoir et du savoir. Ce décalage crée à son tour cette espèce de adiglossie schizophrénique - dont parle Bernard Cerquiglini, et qui peut émerger - le détail n'est pas aléatoire lorsque la langue maternelle d'une minorité est confrontée à l'écriture $^{5}$. En résulte un texte lourd de silences, réduit à son

3 Georges Bélanger, * Portrait d'auteur: Patrice Desbiens *, Francophonie d'Amérique, $\mathrm{n}^{\circ} 2,1992$, p. 95.

4 Patrice Desbiens, L'bomme invisible/The Invisible Man, Sudbury et Moonbeam, Prise de Parole et Penumbra Press, 1981, p. 22. Les citations seront désormais paginées dans le texte.

5 Bernard Cerquiglini, La naissance du français, Paris, Presses universitaires de France, 1991. Voir surtout les pages $47-48$, dans lesquelles il décrit cette - Iolpération des plus complexes, alors qu'on la croit simple, qui implique 
82

expression la plus simple ou la plus grossière, parfois violente, dans tous les cas d'une tragique banalité. De là, aussi, le refus catégorique, de la part de l'auteur, du poétique, de l'intellectualisme, de tout vernis littéraire qui édulcorerait la situation et qui permettrait peut-être aux lecteurs de parcourir ce récit brutal en toute sérénité.

Il n'y a pas, on le sait en cette fin de $x^{\mathrm{e}}$ siècle, de sujet parlant qui ne soit pas toujours déjà lézardé et hétérogène à luimême. Précisons toutefois l'ampleur de cette schize chez le personnage de Desbiens, si sclérosante qu'elle le frappe de mutisme et le réduit aussitôt à un "il * invisible, anonyme, divisé. Avant même l'incipit, l'ensemble des éléments paratextuels contribue à créer l'impression d'un miroir lisse entre les univers francophone et anglophone, depuis les titres équivalents, les marques génériques (*Un récit/A Story *) et la double pagination jusqu'à la publication du livre par deux maisons d'éditions ontaroises. Toujours est-il que cet effet de miroir se brise dès les premières pages. Le texte de Desbiens a ceci d'insolite qu'il est non seulement traduit, pour ainsi dire, par l'auteur lui-même, mais les deux variantes qui le constituent ne coïncident pas parfaitement: dans l'intervalle des récits anglais et français s'accusent des décalages riches en signification. Tandis que quelques événements sont laissés dans l'ombre d'une langue, certains réseaux métaphoriques se dessinent uniquement dans l'autre. Et ce sont précisément ces dérapages lexicaux, ces glissements sémantiques, ces divergences thématiques qui font office de révélateurs dans le texte, qui rendront visible, le temps de la lecture du moins, l'ombre de l'homme invisible.

Le statut d'^inexistence * du héros est rendu sensible dès l'introduction, où se juxtaposent des appartenances que d'aucuns croiraient neutres:

L'homme invisible est né à Timmins, Ontario. / Il est FrancoOntarien.

The invisible man was born in Timmins, Ontario. / He is French-Canadian (p. 1).

que l'on rebâtisse le réseau des signes par lequel on perçoit et affirme le monde. Opération de transfert, en effet, qui attribue à la langue maternelle quelques-unes des valeurs de l'idiome paternel, $[\ldots]$ la langue Imaternellel acquérant un lien au savoir et au pouvoir $[\ldots]$ - p. 47-48. 
La différence du personnage est tour à tour accusée et gommée sous l'effet du rouleau compresseur d'un autre toujours plus fort : tantôt c'est le Franco-Ontarien minoritaire et défavorisé face au Québécois de souche, " pure laine - et majoritaire en sa province; tantôt le "French Canadian " sans spécificité aucune aux yeux du Canadien anglais (ou disons, pour simplifier: du Canadien tout court). S'établit d'entrée de jeu une hiérarchie identitaire constituée d'abord de l'Américain, suivi du Canadien (anglophone), puis du Québecois. Quel que soit le sujet normatif et plein par rapport auquel se définit - ou plutôt est défini, faute de posséder sa propre voix - le Franco-Ontarien, ce dernier sera invariablement ravalé au bas de l'échelle. C'est en fonction de cette hiérarchie de capital culturel (pour emprunter l'expression de Bourdieu) que, par exemple, dans une dernière tentative de fuite, le personnage de la version française encaissera sa prestation d'assurance-chômage pour la changer en chèques de voyage canadiens, alors que son double de la version anglaise, lui, changera sa paie en traveller's cheques américains.

\section{I) Deux poids, deux mesures: le statut des langues dans le récit}

Il ne faut donc pas se leurrer par l'apparente égalité que semble conférer la présence des deux langues dans le texte. Force est de constater que le français et l'anglais n'y ont pas le même poids symbolique. À en juger d'après la facture du récit, le français de l'Ontarien, à lui seul insuffisant, doit être suppléé par l'anglais. Depuis les anglicismes qui traversent de part en part les pages françaises ("une canne de binnes. (p. 39); "Fait sonner le change dans ses poches. (p. 45)), jusqu'à la voix anglophone du mépris que l'homme ne tarde pas à intérioriser et qui le replonge de plus belle dans son silence (“ Dive! Dive! Dive!” crie l'homme invisible à l'équipage de son sous-marin - (p. 23)), la langue maternelle est sans cesse érodée, rendue étrangère à elle-même, par la menace lancinante et omniprésente de l'anglais. Ces fréquentes irruptions de mots anglais ne nécessitent pourtant aucun recours à la traduction. Telle est la réalité du bilinguisme avec laquelle doit forcément composer toute minorité linguistique, règle à laquelle les Franco-Ontariens n'échappent surtout pas. Non sans ironie, cette capacité surdéveloppée d'adaptation devient l'unique trait définitoire du héros invisible - ce qui, à la fois, le différencie de l'autre et le rend parfaitement indifférenciable, "lézard tacheté..." 
doté d'une . langue fourchue et de prodigieux "pouvoirs de caméléon" (p. 40).

La version anglaise, en revanche, est à l'épreuve des incursions du français. Tout se passe comme si le personnage s'exprime en anglais à moins que l'emploi du français ne soit précisé en toutes lettres dans le texte - et cela, même si l'utilisation du français, dans une telle situation, serait une aberration, un acte qui, même pour un francophone natif, s'écarterait de la norme: - He orders a hot hamburger sandwich./Well-done./No onions/ In French" (p. 24). Le texte ne cesse d'entériner ce déséquilibre initial des rapports de pouvoir. Ainsi, seul l'anglais s'arroge le droit de déterminer la valeur réelle des choses. Que l'on compare le déplacement de perspective qui s'effectue presque insensiblement entre l'énoncé qualificatif: :Il commence à parler français de plus en plus * et ces jugements de valeur: "His French starts getting better and better. (p. 22); "They speak a very good French. (p. 24; je souligne).

À toute tentative de porter la voix francophone au chapitre, le texte répondra toujours par un moyen nouveau de l'étouffer, comme si les droits du français à la représentation, soit à l'accès au savoir et au pouvoir, lui étaient d'emblée forclos. On ne s'étonnera donc pas de ce que la transition difficile à l'écriture, marquée par l'indigence du vocabulaire, l'aspect rudimentaire de la syntaxe caractérisée par l'*avalement * d'articles et de prépositions et les fautes d'orthographe ${ }^{6}$, porte les empreintes de l'oralité : - [Le soleil] leur rit dans face. (p. 3); *Il est l'autre bord de la rue. (p. 24); "Attention tassez-vous le vlà qui repart * (p. 25); " Il s'est tiré une balle dans tête..." * (p. 41).

Cette pauvreté linguistique peut confiner ceux qui ont «[1]a langue dans la poche ( (p. 19) à l'aphasie et au mutisme. Voilà que les phrases se répètent trois fois d'affilée dans le récit (p. 26):

6 On retrouve effectivement des erreurs dans le récit de Desbiens - clins d'œil de l'auteur ou coquilles?: * il crit • ou * il pleurt * , fautes d'autant plus révélatrices si l'on considère que l'un des objectifs plus ou moins avoués de la codification, au XVII ${ }^{e}$ siècle, d'une orthographe française alambiquée, aurait été de distinguer l'ignorance de l'élite... (Voir Henriette Walter, Le français dans tous les sens, Paris, Robert Iaffont, 1988). Autre fait intéressant, le texte situe le moment de rupture de l'homme invisible d'avec la langue écrite à l'adolescence, lorsqu'il se désintéresse tout à coup de ses études et, justement, «ne gagne plus de concours d'orthographe - (p. 17). 
le personnage semble bégayer. Elles se bousculent et s'entrechoquent : il balbutie. La langue lui échappe et se volatilise, les mots qu'il * avale * s'enflamment et le brûlent : * Il feuillette le billet allerretour de sa langue./Le billet prend feu et se recroqueville dans le cendrier de sa bouche" (p. 43). Délesté d'un nom (tout comme les pin-ups dans les revues pornographiques qui, s'imagine-t-il, " sont peut-être des Canadiennes françaises qui ont changé de nom " (p. 38)), le héros orphelin *oublie à son tour le nom de son amante, de son pays, si tant est que celui-ci existe réellement. "His pain has no name. (p. 43): c'est là le douloureux conflit qui le torture. Comment communiquer le mal de la langue, quand on ne dispose pas, partant, des mots pour le dire et se dire? Convoqué à un entretien à l'agence d'assistance sociale et mis en demeure de justifier sa situation de chômeur, * [l]'homme invisible ne peut pas répondre. Il a la langue dans poche d'en arrière de ses jeans sales. Il est assis sur sa langue. Elle lui fait mal * (p. 40 fin). Ou d'ironiser plus sèchement encore le narrateur: "He's got a Frog in his throat." (p. 26).

Ce n'est pas dire pour autant que la fissure du locuteur se colmate dès qu'il s'immisce en terrain anglophone. C'est ce que tend à démontrer, d'une part, l'hypertrophie intertextuelle. Les citations (et pas n'importe lesquelles: des bribes de *Yankee Doodle ", des devises de Monopoly, des refrains des Beatles, des réminiscences d'un premier reader d'enfants ${ }^{7}$ ) prolifèrent dans le discours rapiécé de l'homme invisible, à telle enseigne qu'il finit par ressembler à un automate qui se contente de réciter les formules qu'on lui aurait sérinées ${ }^{8}$. Sans compter, d'autre part, que le protagoniste a beau parler la langue majoritaire, personne ne l'écoute, et qui pis est, ne l'entend. À coup sûr, il sera renvoyé de son rôle d'acteur dans un film d'action (emploi qu'il détient uniquement dans la variante anglaise), parce qu'il y est aphone à

7 Par cxemple: $-|H| \mathrm{e}|\ldots|$ goes directly to the liquor store. He does not pass GO. He does not collect two hundred dollars - (p. 35); - Pauline is a FrenchCanadian hippic girl. / She's just seventeen and you know what I mean. (p. 22$) ; \cdot$ See the cat./See the dog./See the cat run- (p. 8), etc.

8 Ce phénomène intertextuel existe également du côté français, par exemple cette référence à un vers de Verlaine: - Les violons saoûlons de l'automne jouent une musique triste dans les yeux de l'homme invisible. (p. 42) ou cette traduction boiteuse de la chanson Like a Rolling Stone de Bob Dylan: "Comment ça file d'être tout seul comme une meule à Timmins Ontario. (p. 16). 
86

son insu: - We've been looking at the rushes and everytime you open your mouth nothing comes out..." (p. 44).

Le récit de Desbiens porte donc en lui-même le déni d'oppositions aussi tranchées que langue publique/langue privée, culture populaire/culture d'enseignement, étant donné la radicale insuffisance de chacune des deux langues et cultures à répondre aux besoins existentiels de la vie. Ni l'une ni l'autre ne parviendra, somme toute, à se mettre au diapason du quotidien francoontarien. D'où le besoin de chercher dans d'autres cultures les moyens de meubler l'expérience humaine. La rencontre de personnages nommés Baudelaire et Rimbaud à l'école primaire SaintAlphonse de Timmins s'inscrit dans une telle perspective. Coiffé à la Dylan, Rimbaud parle anglais, feuillette la revue Time et s'achemine, en bon enfant terrible, vers les États-Unis. Quant à ce flâneur de Baudelaire, il déménagera au Québec, histoire de se forger une identité. Qu'on se le tienne pour dit: dans l'univers que brosse Desbiens, le * devenir-visible * ne se réalise qu'ailleurs, par l'assimilation obligée à l'une des deux cultures majoritaires.

\section{II) World War Two et le Jésus de Timmins : oppositions thématiques}

Bien que l'homme invisible, à la fin du récit en anglais, soit parti pour les États-Unis en autobus, le voilà qui attend au terminus, dans la version française, un autobus qui ne vient toujours pas. Ce retard vaut aussi bien au sens figuré qu'au sens propre. Ainsi, Desbiens remplace l'image des motoneiges ${ }^{9}$, dans la comparaison suivante: "Le temps passe comme des motoneiges dans les yeux de l'homme invisible ", par celle d'automobiles ( like cars ) (p. 20). Et pour peu que le Franco-Ontarien se rattrape (on lira plus loin que :[1]e temps passe comme des voitures et des camions = (p. 34)), voilà que le texte anglais lui aussi s'est accéléré, et que d'ores et déjà le temps s'amenuise - like a flight of World War Two bombers. (p. 34)! Décidément, tout y paraît plus imposant : une "piscine de Coca-Cola * se transforme, dans le miroir grossissant anglais, en * an ocean of Coca-Cola * (p. 10). De semblables mutations s'opèrent chez le personnage; d'élève

9 Notons, par ailleurs, que la version française, tout imprégnće de cet -êtat d'esprit - (p. 2) qu'est l'hiver canadien où il fait toujours au moins - -10 degrés Celsius dehors - (p. 40), met davantage l'accent sur la rigueur du climat. 
compétent qu'il était autrefois, il deviendra invincible dans la version anglaise: "L'homme invisible n'est plus aussi bon à l'école*; "Used to be, the invisible man was invincible in school" (p. 17).

On s'aperçoit rapidement que l'univers francophone est présenté comme arriéré, ni plus ni moins, par rapport à sa contrepartie anglophone. Placée sous le sceau de l'espace rural, du catholicisme, de l'impuissance politique et du refoulement sexuel, la société francophone est en porte-à-faux par rapport à l'univers urbain, séculier, hautement industrialisé et politisé du Canada anglais. Répartition qui sera lourde de conséquences quant à la division du travail narratif: si le français décrit la misère noire, la bureaucratie hostile, le monde humiliant du bien-être social et de l'assurance-chômage, l'anglais, pour sa part, se voit attribuer, en sa qualité de langue de conquête, les caractéristiques de la virilité et du (soi-disant) progrès à travers les images récurrentes de la guerre et du cinéma.

Il importe d'insister, à cet égard, sur les métaphores guerrières filées dans le récit en anglais. Par exemple, lorsque Katerine (ou Catherine, selon le point de vue) quitte l'homme invisible, (rupture qui annonce l'avènement, en français, de "l'ère glaciale "), les séquelles sont tout autres en anglais: "The bullets pierce his body from both sides./It happens so fast that there is no time for peace talks " (p. 31). Il existe, malgré ce symbolisme militaire, une volonté impérieuse de masquer la souffrance dans la version anglaise, où sont escamotées, du reste, les traces du féminin, et où est diminuée, pour ne pas dire totalement occultée, la vulnérabilité de l'homme invisible. "'Sluffer, and make it look funny...' (p. 40), commande le cinéaste à l'acteur. Si bien qu'il faudra attendre la version française, que le mal de vivre infléchit tout entière, pour contrecarrer cette esthétisation de la violence et de la souffrance.

De la rhétorique de la conquête militaire à celle de la conquête sexuelle, le pas sera vite franchi. Mais les exploits sexuels de l'homme invisible perdent de leur éclat dans le texte en français, langue dans laquelle il s'avérerait impossible de chasser la violence, puisqu'en elle surgit, intarissable, la conscience de la douleur portée à l'autre jusque dans l'intimité. Comparons :

At first they don't have a bed, so they fuck on the kitchen floor.

At first their love is so hot it burns the plaster off the walls.

En premier, ils n'ont pas de lit, même pas de matelas, ils font l'amour sur le plancher de la cuisine. Le plancher de la cuisine 
est dur et froid et Katerine pleure souvent sous les affections de l'homme invisible... (p. 30)

L'interdit religieux y serait-il pour quelque chose? Car le tabou sexuel pèse encore, qu'ils le veuillent ou non, sur ces francophones catholiques, de culture sinon de croyance. Témoin, dans la comparaison suivante, la valeur méliorative de l'énoncé en anglais, alors que la version française utilise un vocabulaire péjoratif qui avilit la femme et l'acte sexuel. * Pauline est la fille la plus cochonne que l'homme invisible ait jamais rencontrée " devient "Pauline is the sexiest girl the invisible man has ever met " (p. 22). Cette emprise encore tenace de l'Eglise sur les habitants de la ville franco-ontarienne paraît pour le moins étouffante. Au film d'action hollywoodien correspond, mutatis mutandis, le spectaculaire du catholicisme, "une comédie musicale [où] la musique joue fort " (p. 9). Au contraire de l'anglais, les références à la religion ponctuent systématiquement le texte français ${ }^{10}$, le plus souvent sur le mode du blasphème - la valeur provocatrice de ces propos ne déployant ses pleins effets, bien entendu, que dans l'espace francophone. N'empêche que le lexique religieux dépasse la simple fonction du juron explétif ("Christ qu'y fait frette! [...] Christ de pays sale !* (p. 35)) pour faire figure d'intertexte important dans la narration française. Outre la mise en scène parodique d'une rivalité entre l'homme invisible et Jésus (cependant né dans une grange à Timmins, de parents ambitieux et nantis), la variante française est parsemée çà et là d'allusions bibliques blasphématoires: *C'est la première job de l'homme invisible. Job, rappelonsnous, c'est le nom du gars dans la Bible qui s'est fait chier dessus par Dieu. Dieu c'est pas juste un pigeon. - (p. 28).

Nul doute que le langage grossier et scatologique qu'emploie l'homme invisible se veut un rempart contre la violence symbolique qui l'assène au jour le jour, une dernière tentative de rescaper un pouvoir, ne serait-ce que le pouvoir de choquer. Il n'en demeure pas moins un important indicateur de son impuissance et de son humiliation. Ainsi les prestations d'assurance-chômage qu'il reçoit sont assimilées à de -petites crottes brunes, [tombées] directement du trou d'cul du gouvernement. (p. 40 s.). L'abjection, la honte et le dégoût de soi occupent désormais toute la

10 Par exemple, alors que ses - best friends start coming to serenade Catherine * (p. 30), il s'agira ailleurs d'- un chour d'hommes très visibles lquil vient chanter des alléluias. (p. 31) à son amante Katerine. 
place du quotidien. It n'est que de se rappeler, pour s'en convaincre, la métaphore qui transforme l'homme invisible en cafard baignant dans une cuve de toilettes: "Et l'homme invisible, dansant comme une coquerelle dans le tourbillon d'eau brune, disparaitra dans les tuyaux rouillés de la ville. "(p. 40 fin).

À la déclaration aux accents épiques sur laquelle s'achevait Maria Chapdelaine, à savoir "[cles gens sont d'une race qui ne sait pas mourir "', Patrice Desbiens riposte, dans L'bomme invisible/ The Invisible Man, par l'illustration d'un personnage d'une autre engeance qui, dirait-on, ne serait bonne qu'à cela: "Hey, you sure know how to die!..." lui dit un de ses amis./L'homme invisible, immédiatement flatté, se fait tirer et meurt souvent./Ce n'est que le commencement... (p. 6). Sans passé, sans avenir, le temps de l'écriture file vers la mort de l'homme invisible. Pendant quelques semaines seulement, grâce au travail, à l'alcool, à une amante, il était * dev[enu] presque normal* (p. 28), "si près d'être visible. (p. 31), - trop bref intermède, hélas, à la suite duquel il ne lui restera plus que l'alcool, et l'attrait du suicide auquel il doit chaque jour résister. De Timmins à Québec en passant par Toronto, il se résigne à *se prom[ener] visiblement le long des rues. C'est ça qu'on fait quand on est sur le bien-être..." (p. 27). Errant, anonyme, apatride, l'homme invisible se voit partout réfléchi dans les paysages qu'il parcourt. Mais dans ces reflets, il se découvrira profondément étrange, voire étranger à lui-même. En cela, il est façonné à l'image du texte dont les deux versions fonctionnent, en regard l'une de l'autre, on l'a vu, à la manière d'un miroir déformant, où le sens, éternel nomade, se trace et s'efface, avance puis recule sur la surface mouvante de l'écriture.

L'aliénation que connaît le personnage principal en mal d'origine nous est communiquée dans l'expérience vertigineuse de la lecture, à travers ce redoublement textuel qui déboute coup sur coup chacune de nos attentes. À force de se décupler, la présence surdéterminée des doubles dans le texte fait s'écrouler les distinctions vacillantes entre le dehors et le dedans, le même et l'autre. On se demande, du coup, à quoi pourrait bien ressembler "l'intériorité " de l'homme invisible? Quelle serait son "authentique langue maternelle? Autant d'interrogations grevées d'ambiguités que fait surgir à point nommé la scène suivante:

11 Louis Hémon, Marie Chapdelaine, Montréal, Boréal Express, 1983, p. 198. 
90

"I'd like to fuck them all!... * crie l'homme invisible dans sa langue maternelle.

[...]

"Je voudrais toutes les fourrer !... crie l'homme invisible dans sa langue maternelle.

Il court aux toilettes et vomit violemment.

.I'd like to fuck them all!... screams the invisible man in his mother tongue.

(p. 39)

À la vérité, c'est rien moins que la notion de langue maternelle qui est donnée comme caduque dans cette problématique trouble du bilinguisme franco-ontarien. C'est ce que semble véritablement "traduire "le double récit de Desbiens : la douleur de parler une langue qui est dans tous les sens du terme abâtardie, et la dépossession d'un être humain renvoyé à son propre silence. 